\title{
Kein Einfluss auf die Krankheitsprogression
}

Fragestellung: Hat Pioglitazon einen neuroprotektiven Effekt in der Frühphase der Parkinson-Erkrankung?

Hintergrund: Pioglitazon ist als Insulinsensitizer in der Therapie des Diabetes mellitus Typ 2 etabliert. Tierversuche erbrachten Hinweise auf einen möglichen neuroprotektiven Effekt des Peroxisom-Proliferator-aktivierten-RezeptorGamma-(PPAR $\gamma$-) Agonisten.

Patienten und Methoden: Insgesamt 210 Patienten mit einem innerhalb der letzten fünf Jahre diagnostizierten idiopathischen Parkinson-Syndrom und einer gleichbleibenden Therapie mit Rasagilin (1 mg/d) oder Selegilin (10 mg/d) wurden in diese multizentrische Phase-II-Studie eingeschlossen und $\mathrm{zu}$ einer Therapie mit $15 \mathrm{mg} / \mathrm{d}$ Piaglitazon, $45 \mathrm{mg} / \mathrm{d}$ Pioglitazon oder Placebo in einem Verhältnis von $1: 1: 1$ randomisiert. Nach 44 Wochen wurde der primäre Endpunkt, die Veränderung des Gesamtscores der Unified Parkinson's Disease Rating Scale (UPDRS), analysiert. Sekundäre Endpunkte umfassten Veränderungen der Lebensqualität (Parkinson's Disease Questionnaire 39), der Stimmung (Geriatrische Depressionsskala) und der Kognition (Demenzskala).

INDS Exploratory Trials in Parkinson Disease (NET-PD) FSZONE Investigators. Pioglitazone in early Parkinson's disease: a phase 2, multicentre, doubleblind, randomised trial. Lancet Neurol 2015; 14: $795-803$
Ergebnisse: In der Auswertung des primären Endpunktes zeigte sich nach 44 Wochen eine mittlere Zunahme des UPDRS-Gesamtscores von 4,42 Punkten für 15 mg/d Pioglitazon, von 5,13 Punkten für 45 mg/d Piogli- tazon und von 6,25 Punkten für Placebo ( Abb. 1). Hieraus ergibt sich eine mittlere Differenz von - 1,83 Punkten ( $80 \%$-Konfidenzintervall [KI]: $-3,56$ bis - 0,1) zwischen $15 \mathrm{mg} / \mathrm{d}$ Pioglitazon und Placebo und von - 1,12 Punkten (80\%-KI - 2,93 bis 0,69 ) zwischen $45 \mathrm{mg} / \mathrm{d}$ Pioglitazon und Placebo, sodass für beide Dosierungen kein bedeutsamer Unterschied nachweisbar war $(\mathrm{p}=0,19$ und $\mathrm{p}=0,09)$. Weiterhin erbrachte die Auswertung der sekundären Endpunkte keine signifikanten Unterschiede. Die insgesamt 18 schweren unerwünschten Ereignisse (6:9:3) wurden als nicht therapieassoziiert bewertet.

Schlussfolgerung: Pioglitazon hat höchstwahrscheinlich keinen Effekt auf die Krankheitsprogression in der Frühphase der Parkinson-Erkrankung.

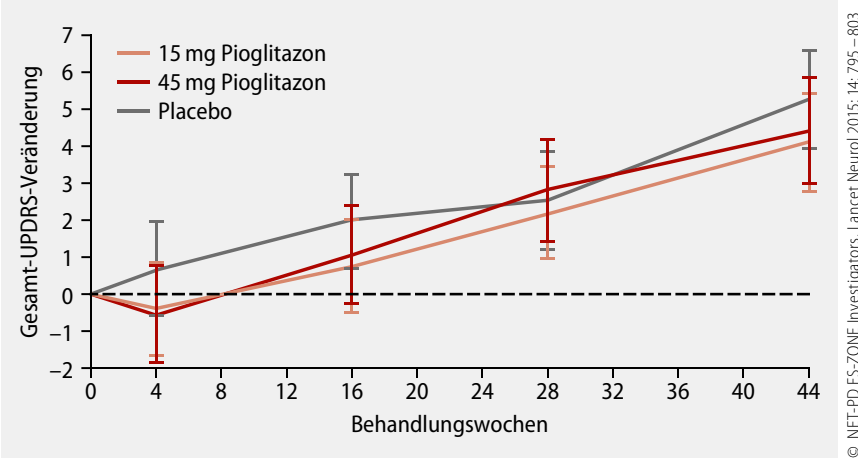

1 Keine signifikanten Unterschiede zwischen den Behandlungsgruppen im Hinblick auf die Änderung der UPDRS im Zeitverlauf.

\section{- Kommentar von Steffen Paschen und Günther Deuschl, Kiel}

\section{Ein neuroprotektiver Effekt von Pioglitazon dürfte damit „Vom Tisch" sein}

Die vorliegende Studie untersucht systematisch mögliche neuroprotektive Effekte beziehungsweise die Wirksamkeit und Verträglichkeit von Pioglitazon in der Frühphase der Parkinson-Erkrankung. Die Auswertung des primären Endpunktes, die Veränderung des Gesamt-UPDRS, zeigte keinen bedeutsamen Unterschied, sodass ein neuroprotektiver Effekt des Antidiabetikums unwahrscheinlich ist. Weitergehende Studien mit dieser Substanz werden deshalb auch nicht empfohlen. Auch für Pioglitazon zeigt sich somit eine Diskrepanz zwischen den vielversprechenden Ergebnissen bei Nagern und Primaten und dem Effekt bei Patienten mit einem idiopathischen Parkinson-Syndrom. Die Studie ist Teil der systematischen Suche nach neuroprotektiven Substanzen von NET-PD, einer amerikanischen vom NIH-geförderten Initiative. Keiner der mitgeführten Outcome-Parameter (dazu gehören auch noch nicht publizierte Biomarker) zeigt ein besseres Ergebnis für die Pioglitazonbehandlung als für Placebo. Natürlich bleibt immer die Frage, ob man mit diesen Therapien zu spät kommt, da der subklinische Krankheitsprozess schon zu weit fortgeschritten ist. Auch andere Faktoren könnten das Ergebnis verfälschen. Es ist aber sehr unwahrscheinlich, dass bei einem so homogen negativen Ergebnis für alle Outcome-Parameter ein Erfolg verpasst wurde.

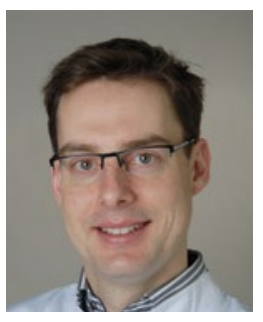

Dr. med. Steffen Paschen, Kiel

Klinik für Neurologie, Universitätsklinikum Schleswig-Holstein, Campus Kiel (UKSH) E-Mail: s.paschen@neurologie.uni-kiel.de 